\title{
Metatranscriptomics provides closer diversity and composition estimates with morphology than PCR-based methods: a zooplankton mock community case study
}

\author{
Mark Louie Lopez ${ }^{1}$, Ya-Ying Lin ${ }^{2}$, Mitsuhide Sato ${ }^{3}$, Fuh-Kwo Shiah ${ }^{4}$, Chih-hao Hsieh ${ }^{5}$, \\ and Ryuji Machida ${ }^{2}$ \\ ${ }^{1}$ Biodiversity Program, Taiwan International Graduate Program, Academia Sinica \\ ${ }^{2}$ Academia Sinica \\ ${ }^{3}$ Nagasaki University \\ ${ }^{4}$ Academia Sinica, Environmental Change Research Center \\ ${ }^{5}$ Institute of Oceanography
}

December 1, 2020

\begin{abstract}
Studying complex metazoan communities requires taxonomic expertise and laborious work if done using the traditional morphological approach. Nowadays, the popular use of molecular-based methods accompanied by massively parallel sequencing (MPS) provides rapid and higher resolution diversity analyses. However, diversity estimates derived from the molecular-based approach can be biased by the co-detection of environmental DNA (eDNA), pseudogene contamination, and PCR amplification biases. Here, we constructed microcrustacean zooplankton mock communities to compare species diversity and composition estimates from PCR-based methods using genomic (gDNA) and complementary DNA (cDNA), metatranscriptomic transcripts, and morphology data. Mock community analyses show that gDNA mitochondrial cytochrome c oxidase I (mtCOI) amplicons inflate species richness due to environmental and nontarget species sequence contamination. Significantly higher amplicon sequence variant (ASV) and nucleotide diversity in gDNA amplicons than cDNA indicated the presence of putative pseudogenes. Last, PCR-based methods failed to detect the most abundant species in mock communities due to priming site mismatch. Overall, metatranscriptomic transcripts provided estimates of species richness and composition that closely resembled morphological data. The use of metatranscriptomic transcripts was further tested in field samples. The results showed that it could provide consistent species diversity estimates among biological and technical replicates while allowing monitoring of the zooplankton temporal species composition changes using different mitochondrial markers. These findings show that community characterization based on metatranscriptomic transcripts reflects the actual community more than PCR-based approaches.
\end{abstract}

\section{Hosted file}

Metatranscriptomics20201119.pdf available at https://authorea.com/users/380416/articles/ 496416-metatranscriptomics-provides-closer-diversity-and-composition-estimates-withmorphology-than-pcr-based-methods-a-zooplankton-mock-community-case-study 